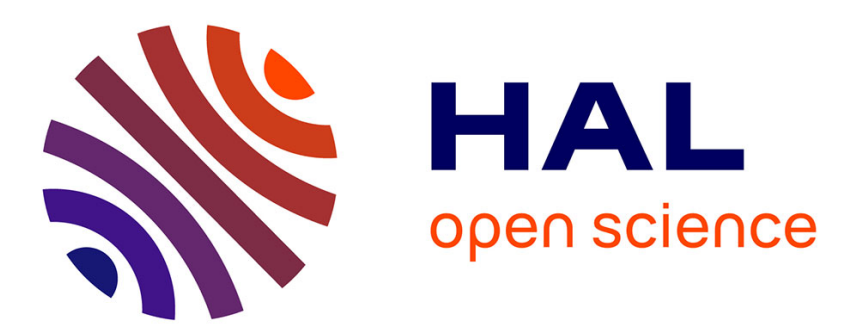

\title{
Hierarchy of the Velocity Structure Functions in Fully Developed Turbulence
}

\author{
G. Ruiz Chavarria, Christian Baudet, R. Benzi, S. Ciliberto
}

\section{To cite this version:}

G. Ruiz Chavarria, Christian Baudet, R. Benzi, S. Ciliberto. Hierarchy of the Velocity Structure Functions in Fully Developed Turbulence. Journal de Physique II, 1995, 5 (4), pp.485-490. 10.1051/jp2:1995144 . jpa-00248174

\section{HAL Id: jpa-00248174 https://hal.science/jpa-00248174}

Submitted on 1 Jan 1995

HAL is a multi-disciplinary open access archive for the deposit and dissemination of scientific research documents, whether they are published or not. The documents may come from teaching and research institutions in France or abroad, or from public or private research centers.
L'archive ouverte pluridisciplinaire HAL, est destinée au dépôt et à la diffusion de documents scientifiques de niveau recherche, publiés ou non, émanant des établissements d'enseignement et de recherche français ou étrangers, des laboratoires publics ou privés. 
Classification

Physics Abstracts

$4725 \mathrm{C}-05.40$

\title{
Short Communication
}

\section{Hierarchy of the Velocity Structure Functions in Fully Developed Turbulence}

\author{
G. Ruiz Chavarria $\left({ }^{1, *}\right)$, C. Baudet $\left({ }^{1}\right)$, R. Benzi $\left({ }^{2}\right)$, and S. Ciliberto $\left({ }^{1}\right)$ \\ ( $\left.{ }^{1}\right)$ Ecole Normale Supérieure de Lyon, Laboratoire de Physique $\left({ }^{* *}\right), 46$ Allée d'Italie, 69364 \\ Lyon, France \\ $\left({ }^{2}\right)$ Dipartımento di Fisica, Universitá dı Roma, "Tor Vergat", Via della Ricerca Scıentifica 1, \\ 00133 Roma, Italy
}

(Received 19 September 1994, revised 25 January 1995, accepted 6 February 1995)

\begin{abstract}
Experimental evidence is given that the hierarchy for the energy dissipation moments recently proposed by She and Leveque (1994) for fully developed turbulence can be generalized to the velocity structure functions. The scaling extends down to the Kolmogorov length.
\end{abstract}

To characterize the statistical properties of fully developed turbulence, the moments $S_{n}(r)$ of velocity differences on the scale $r$ are of special interest, specifically:

$$
S_{n}(r)=<|V(x+r)-V(x)|^{n}>
$$

where $\langle$. $>$ stands for ensemble average and $V$ is the velocity component parallel to $r$. At high Reynolds number $R e=U_{0} \cdot L / \nu$ the $S_{n}(r)$ satisfies the relation

$$
S_{n}(r) \propto r^{\zeta(n)}
$$

for $L>r>>\eta$ where $L$ is the integral scale, $\eta=\left(\nu^{3} / \epsilon\right)^{1 / 4}$ is the dissipative scale, $\epsilon$ is the energy dissipation rate, $\nu$ the kinematic viscosity and $U_{0}$ the mean velocity of the flow. The range of length $L>r>>\eta$ where the above-mentioned power law is satisfied is called the inertial range The Kolmogorov (K41) theory [2] predicts $\zeta(n)=n / 3$, but experimental [3] and numerical [4] results show that $\zeta(n)$ deviates substantially from the linear law at large $n$. This phenomenon is believed to be produced by the intermittent behaviour of the energy dissipation [5] which can be taken into account by rewriting equation (1) in the following way:

$\left({ }^{*}\right)$ On leave from Departemento de Fisica, Facultad de Ciencias, UNAM, Ciudad Universitaria, 04510 Mexico D.F., Mexico

$\left({ }^{*}\right)$ CNRS URA 1325

(C) Les Editions de Physique 1995 


$$
S_{n}(r) \propto<\epsilon_{r}^{n / 3}>r^{n / 3} \propto r^{\tau(n / 3)+n / 3}
$$

where $\epsilon_{r}$ is the average of the local energy dissipation $\epsilon(x)$ on a volume of size $r$ centered around a point $x$. A comparison of equations (1) and (2) leads to the conclusion that the scaling exponents $\tau(n)$ of the energy dissipation are related to those of $S_{n}$ by $\zeta(n)=\tau(n / 3)+n / 3$. Since the Kolmogorov (K61) theory [5] many other models [6-10] have been suggested to describe the behaviour of $\zeta(n)$. However, several of these models appeal to some specific hypotheses which cannot be directly checked.

In a very recent letter [11] She and Leveque have proposed an interesting theory to explain the anomalous scaling exponents of velocity structure functions. The theory yields a prediction

$$
\zeta(n)=n / 9+2\left(1-(2 / 3)^{n / 3}\right)
$$

which is in a very good agreement with the available experimental data $[9,11,12]$.

In order to get this result they made a fondamental assumption on the hierachy of the moments of the local energy dissipation. Specifically they assume

$$
\frac{\left\langle\epsilon_{r}^{n+1}>\right.}{\left.<\epsilon_{r}^{n}\right\rangle}=A_{n}\left(\frac{<\epsilon_{r}^{n}>}{<\epsilon_{r}^{n-1}>}\right)^{\beta}\left(\epsilon_{r}^{(\infty)}\right)^{(1-\beta)}
$$

where $A_{n}$ are geometrical constants and $\epsilon_{r}^{(\infty)}=\lim _{n \rightarrow \infty} \frac{\left\langle\epsilon_{r}^{n+1}\right\rangle}{\left\langle\epsilon_{r}^{n}\right\rangle}$ is associated in reference [11] with filamentary structures of the flow. On the basis of simple arguments, it is assumed [11] that: $\epsilon_{r}^{(\infty)} \propto r^{-2 / 3}$ and $\beta=2 / 3$. More recently in two independent papers Dubrulle [13] and She [14] have shown that equation (4) is a direct consequence of a log-Poisson distribution of the energy dissipation rate. Equation (4) has been verified numerically [15] and experimentally [16]. From an experimental point of view the calculation of $\epsilon_{r}$ is certainly more complicated than that of the $S_{n}$. It is therefore interesting to understand whether a hierarchy of moments like equation (4) also holds for the velocity structure functions. According to equation (2) this could indeed be the case. Let us, therefore, suppose that the following log-Poisson hierarchy is true:

$$
\frac{S_{p+1}}{S_{p}}=B_{p}\left(\frac{S_{p}}{S_{p-1}}\right)^{\beta^{\prime}}\left(S_{(\infty)}\right)^{\left(1-\beta^{\prime}\right)}
$$

where $B_{p}$ are geometrical constants and $S_{(\infty)}=\lim _{p \rightarrow \infty} \frac{S_{p+1}}{S_{p}}$. It is very simple to derive the following equation from equation (4):

$$
\frac{S_{p+3}}{S_{p}}=C_{p}\left(\frac{S_{p}}{S_{p-3}}\right)^{\gamma}\left(S_{(\infty)}\right)^{3(1-\gamma)}
$$

where $\gamma=\left(\beta^{\prime}\right)^{3}$. The consequence of equation (6) for $p=3$ is

$$
\left(S_{(\infty)}\right)^{3(1-\gamma)} \propto \frac{S_{6}}{S_{3}^{(1+\gamma)}}
$$

Using equation (2) in equations (6) and (7) one immediately recover equation (4) with $\beta=\gamma$ and $n=p / 3$. We rewrite equations (5) and (7) in a more synthetic form:

$$
F_{p+1}(r)=C_{p}\left(F_{p}(r)\right)^{\beta^{\prime}} \tilde{F}(r)
$$


where

$$
F_{p+1}(r)=\frac{S_{p+1}(r)}{S_{p}(r)}
$$

and

$$
\tilde{F}(r)=\left(\frac{S_{6}}{S_{3}^{(1+\beta)}}\right)^{\frac{\left(1-\beta^{\prime}\right)}{3(1-\beta)}}
$$

The purpose of this communication is to verify equation (8) experimentally not only at high $R e$, where an inertial range exists, but also at small $R e$, where no inertial range is detectable.

Let us remark that at low $R e$, we can still compute the scaling exponents of the structure functions, by using the notion of Extended Self Similarity (ESS) [12], i.e. we compute the scaling of $S_{p}$ against $S_{3}(r)$ :

$$
S_{p}(r) \propto\left[S_{3}(r)\right]^{\zeta(p)}
$$

where $\zeta(p)$ are given by equation (3). In our experiments, equation (10) is verified for $8 \eta<$ $r<L$ [12]. Thus we expect that, a prori, equation (8) should also be satisfied, at least in the same range. However, as we shall see below, equation (8) is found to hold for any $r<L$, even for $r<\eta$.

The experimental apparatus has been described in detail elsewhere $[12,13]$ and we report here just the main features. It consists of a wind tunnel with a cross-section $0.5 \times 0.5 \mathrm{~m}^{2}$ and a length of $3 \mathrm{~m}$. Turbulence is produced with a grid, a cylinder and a jet. The integral scale $L$, that is the grid spacing, the cylinder diameter and the jet hole diameter is changed from 5 to $10 \mathrm{~cm}$ in order to change $R e$ without changing the speed. The mean velocity is varied in the range $1 \mathrm{~m} / \mathrm{s}<U_{0}<8 \mathrm{~m} / \mathrm{s}$ consequently with this arrangement $R e$ can be set in the range $4000<R e<300000$. The corresponding $R_{\lambda}$, the Reynolds number based on the Taylor microscale [1], is in the range $100<R_{\lambda}<800$. The instantaneous velocity is measured with a hot wire connected to a TSI constant temperature anemometer. The hot wire has a diameter of $50 \mu \mathrm{m}$ and a length of $0.25 \mathrm{~mm}$. The velocity probe was placed more than $25 \mathrm{~L}$ downstream the object producing turbulence. At this distance, downstream turbulence can be considered locally homogeneous and isotropic, as we have discussed in several papers [12]. The instantaneous velocity signal was digitized with a 16 bits a/d converter. Time series of about $10^{7}$ points have been recorded which allows us to compute with a statistical accuracy of a few percent up to the $9^{\text {th }}$ order structure function. The local time measurements are transformed into spatial measurements by using the Taylor hypothesis, that is $x=U_{0} \times t$.

In this communication we will use several data sets with $R_{\lambda}$ ranging from 140 to 800 . In this way one can compare data at high $R e$, where an inertial range is present, with data at small $R e$, where the inertial range is not yet developed. This can be seen in Figure 1(a) where $S_{6}(r) / r^{\zeta(6)}$, computed for two experiments at $R_{\lambda}=800$ and $R_{\lambda}=140$ with $\zeta(6)=1.78$ [12], is reported. The plateau, corresponding to the inertial range, is well-defined only for the jet data. In Figure 1(b) we also show $S_{3} / r$ for the same $R_{\lambda}$ as in Figure 1(a).

Using the $S_{n}(r)$ computed from our data, we are able to check equation (8) with $\beta=2 / 3$, which is the value predicted by She and Leveque, and which has been found both experimentally and numerically. The result is shown in Figure (2), where the function $F_{p+1}(r)$ is plotted as a function of $\left[F_{p}(r)\right]^{\beta^{\prime}} \cdot \tilde{F}(r)$ for several $p$ and different $R e$. We see that the data are aligned on straight lines of slope $1.00 \pm 0.005$, showing that equation (8) is satisfied. As a consequence, this shows that equation (8) is well verified by the experimental data for any $r<L$. This means that the constant $C_{p}$ has the same value in the inertial and in the dissipative range. Furthermore we want to stress that all curves in Figure 2 have been vertically shifted in order to 

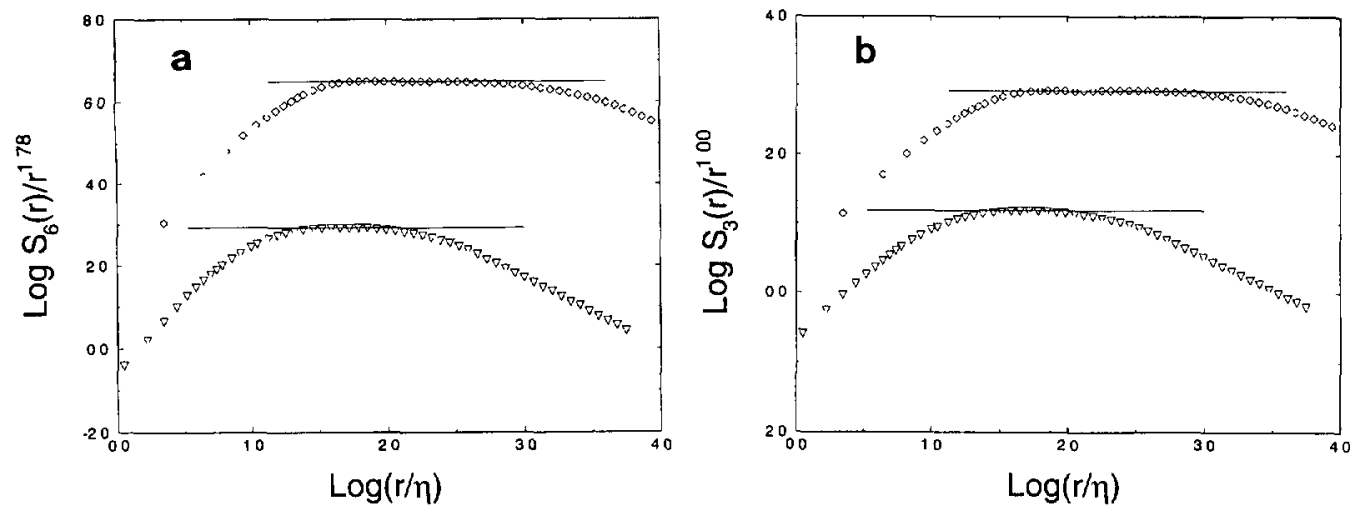

Fig 1. - Structure functions $S_{p}(r) / r^{\zeta(p)}$ as a function of $r$ at $R_{\lambda}=800(\diamond)$ and $R_{\lambda}=140(\nabla)$. (a) $p=6$ and $\zeta(p)=1.78$ (b) $p=3$ and $\zeta(p)=1$. Logarithms are base 10 .
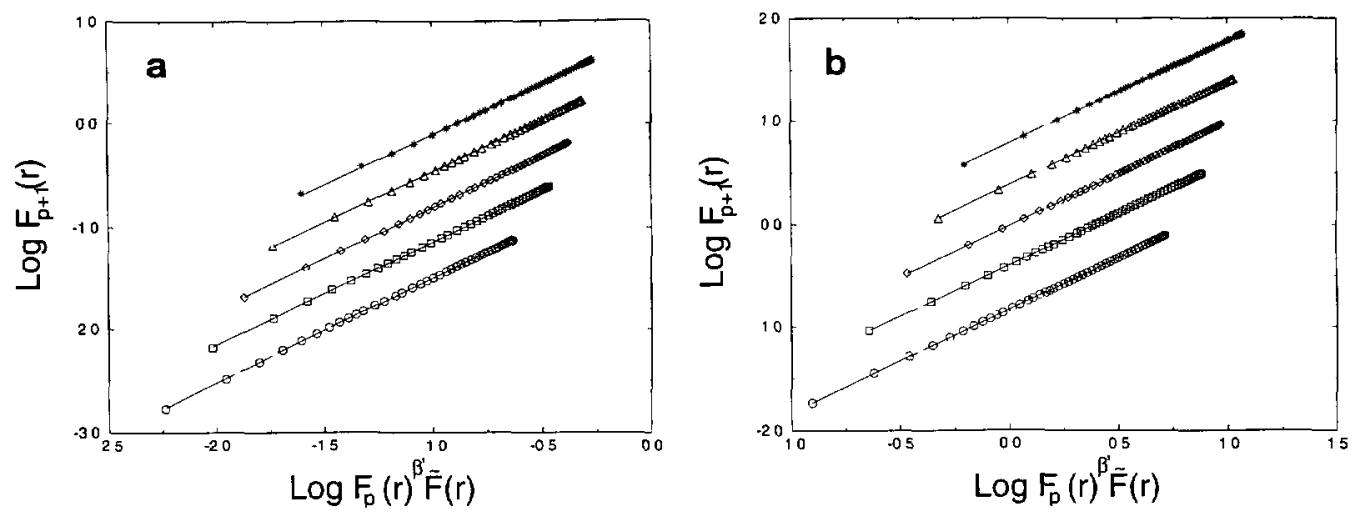

Fig. 2 - The function $F_{p+1}(r)$ defined in equation (8) is plotted, for several values of $p$, as a function of $\left[\left(F_{p}(r)\right)^{\beta^{\prime}} \cdot \tilde{F}(r)\right]$ with $\beta=2 / 3, \beta^{\prime}=\beta^{\delta}$ and $\delta=1 / 3 \quad R_{\lambda}=140$ in (a) and $R_{\lambda}=800$ in (b). The 5 curves in (a) and (b) correspond to $p=1,2,3,4,5$ starting from the bottom lines. They have been vertically shifted of $-0.4,-0.2,0,02,0.4$ in order to separate them. The solid lines have slope 1 . Logarithms are base 10

separate them, otherwise they would perfectly superposed. Thus the constant $C_{p}$ in equation (8) is $p$ independent too. It only depends on $R e$ and on the geometry.

We now compare this result with ESS. In order to do that, using the same data set as that shown in Figure 1, we report the structure functions $S_{6}$ as a function of $S_{3}$ in Figure 3 . We observe a very good scaling for $r>5 \eta$, whereas in Figure 2 we have seen that the scaling of equation (8) holds even for $r<5 \eta$

Finally, we have checked whether $\beta$ depends on Re. Actually, motivated by the experimental work of Castaing et al. [18], Dubrulle suggests in a very recent paper [13] that the exponent $\beta$ could slowly increase as a function of $\log (R e)$. We have checked this hypothesis by considering $\beta$ in equation (8) as a free parameter to be found with a best fit. No appreciable variation is observed as a function of $R e$. The mean value of the exponent $\beta$ is $2 / 3 \pm 0.06$, thus with the accuracy of our data no trend of this exponent as a function of $R e$ has been observed. However, this is a very delicate subject which is still under study. 

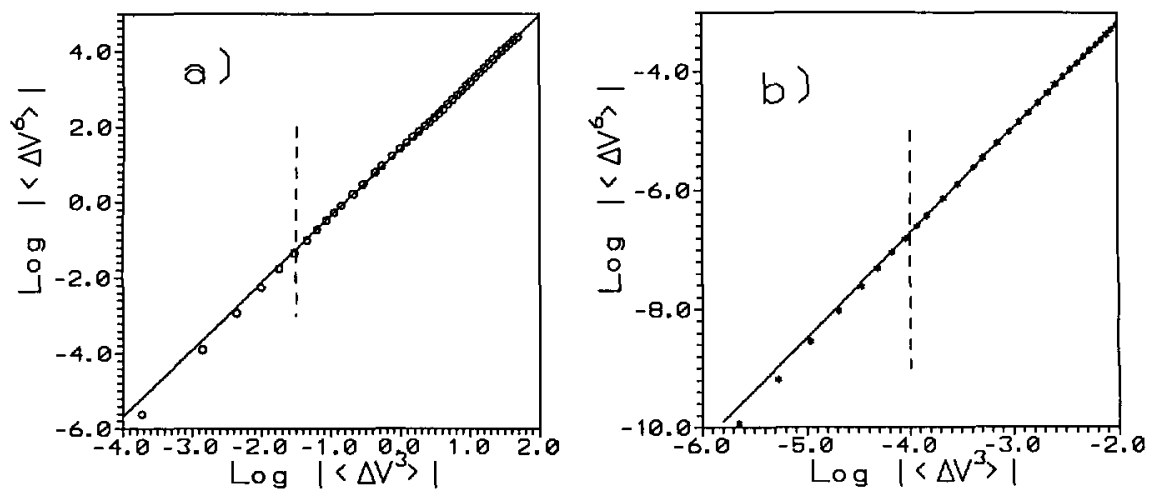

Fig. 3. - Structure functions $S_{6}$ as a function of $S_{3}$ at $R_{\lambda}=800$ (a) and $R_{\lambda}=140$ (b). Vertical dashed lines indicate the value of $S_{3}$ at $5 \eta$. Logarithms are base 10 .

As a conclusion we have experimentally shown that there exists a hierarchy (Eq.(5)) of the velocity structure functions which is related to the hierarchy proposed by She and Leveque [11] for the energy dissipation. The most important result is that equation (5) is valid for any $r<L$ even at scales where dissipation effects are usually thought to become relevant. Furthermore, the fact that this relationship is valid at both high and small $R e$ numbers suggests that this is a general property of structure function in fully developed turbulence.

\section{Acknowledgments}

We acknowledge useful discussions with B. Castaing.

\section{References}

[1] Monin A S. and Yaglom A M, Statıstıcal Fluid Mechanıcs (MIT Press, Cambridge, MA, 1975).

[2] Kolmogorov A.N., C R. Acad. Sci. USSR 30 (1941) 299.

[3] Anselmet F., Gagne Y., Hopfinger E.J. and Antonia R.A., J. Flund Mech. 140 (1984) 63.

[4] Vincent A. and Meneguzzi M., J. Flurd Mech 225 (1991) 1, Briscolin1 M, Santangelo P, Succ1 S. and Benzi R., Phys. Rev. E 50 (1994) 1745

[5] A. N. Kolmogorov, J. Flurd Mech. 13 (1962) 83.

[6] Frish U, Sulem P and Nelkın M., J. Fluıd Mech 87 (1978) 719.

[7] Benzi R., Paladin G., Parısi G. and Vulpianı A, J. Phys. A 17 (1984) 3521.

[8] Meneveau C. and Sreenivasan K.R, Phys. Rev. Lett. 59 (1987) 1424.

[9] Gagne Y and Castaing B., C.R. Acad Scr. Parıs 312 (1991) 441.

[10] She Z S. and Orszag S , Phys. Rev. Lett. 66 (1991) 1701.

[11] She Z.S. and Leveque E, Phys Rev. Lett. 72 (1994) 336

[12] Benzi R., Clliberto S, Tripiccione R., Baudet C. and Succi S , Phys Rev. E 48 (1993) 29; Benzi R., Culiberto S., Baudet C., Ruiz Chavarria G, and Tripiccione R., Europhys. Lett 24 (1993) 275; Benzi R, Cıliberto S., Baudet C. and Ruiz Chavarrı G., Physıca D, to be published in the March 1995 issue. 
[13] Dubrulle B., Phys. Rev. Lett. 73 (1994) 959.

[14] She Z.S. and Waymire E., Phys. Rev. Lett. 73 (1994) in press.

[15] Benzi R. and Biferale L., in preparation.

[16] Ruiz Chavarria G., Baudet C. and Ciliberto S., Phys. Rev. Lett., to be published.

[17] Baudet C., Ciliberto S. and Phan Nhan Tien, J. Phys 3 (1993) 293.

[18] Castaing B. and Gagne Y., Physıca D 46 (1990) 177; Chabaud R, Naert A., Peinke J., Chillá F., Castaing B. and Hébral B., Phys. Rev. Lett. 73 (1994) 3227. 